\title{
Interacting Rényi holographic dark energy with parametrization on the interaction term
}

\author{
Umesh Kumar Sharma ${ }^{1}$, Vipin Chandra Dubey ${ }^{2}$ \\ ${ }^{1,2}$ Department of Mathematics, Institute of Applied Sciences and Humanities, GLA University \\ Mathura-281 406, Uttar Pradesh, India \\ ${ }^{1}$ E-mail: sharma.umesh@gla.ac.in. \\ ${ }^{2}$ E-mail: vipin.dubey@gla.ac.in
}

\begin{abstract}
In the present work, we study the Rényi holographic dark energy model (RHDE) in a flat FRW Universe where the infrared cut-off is taken care by the Hubble horizon and also by taking three different parametrizations of the interaction term between the dark matter and the dark energy. Analysing graphically, the behaviour of some cosmological parameters in particular deceleration parameter, equation of state (EoS) parameter, energy density parameter and squared speed of sound, in the process of the cosmic evolution, is found to be leading towards the late-time accelerated expansion of the RHDE model.
\end{abstract}

Keywords: RHDE, interaction, cosmology

PACS: 98.80.Es, 95.36.+x, 98.80.Ck

\section{Introduction}

Our Universe is undergoing accelerated expansion which is marked by various cosmological observations like type-Ia supernova [1-4], the large-scale structure [5] 8 , cosmic microwave background (CMB) anisotropies [9 11]. For explaining this accelerated expansion of the cosmos the concept of dark energy (DE) was incorporated which is an extraordinary component with negative pressure [12,13. The late-time acceleration of the Universe can be explained by two categories models. First one is dynamical dark energy models in which we change the matter part of the Einstein field equation. Amongst a lot of theories and models the cosmological constant model is the simplest model, initially proposed by Einstein [14 18], which suggests that the equation of the state parameter (EoS) $\omega=-1$ and the cosmological constant is the most basic candidate for dark energy, and it is consistent with observations, besides the finetuning and coincidence problem [15, 19,20]. To get relief from such problems, many dynamical dark energy (DE) models are given, for example, $k$-essence [21], quintessence [22, 23], Chaplygin gas [24], phantom [25], tachyon [26], holographic dark energy (HDE) 27] and new agegraphic dark energy (NADE) [28]. Secondly, by modified gravity theories such as $f(R)$ theory [29], $f(T)$ theory [30], Hořava-Lifshitz gravity [31 34], Brans-Dicke theory [35], Gauss-Bonnet theory [36] and $f(\overline{R, T})$ theory [37], which are obtained by changing the geometric part of Einstein field 
equation. It is shown that within mimetic gravity, a theory of modified gravity which has gained a lot of interest recently, the coincidence problem is alleviated [38. The feasible solution of the cosmic coincidence problem can also be found by taking the interaction between the dark matter and the dark energy [39]. Also, more generally regarding interacting dark energy models, these models can have important observational consequences, particularly for solving the well-known $H_{0}$ tension [40,41].

The HDE has the number of significant features of the quantum gravity and has the traits of holographic principle [42, 43], which states that degrees of freedom (the actual entropy of a system that has to be stored on some boundary in a holographic scenario) are dependent on bounding area instead of volume. The reason for the flat FRW Universe was not known when HDE was considered in terms of Benkenstein entropy using infrared cut-off with the Hubble horizon [44-46]. Physicists have taken various entropies with different cut-off scales like the interaction between cold dark matter and dark energy or combination of the mentioned approaches 47, 48.

In the literature 27, 49 51], HDE model has been considered widely and examined as $\rho_{D} \propto \Lambda^{4}$, while relation between the IR cut-off L, UV cut-off $\Lambda$ and the entropy $\mathrm{S}$ is $\Lambda^{3} L^{3} \leq(S)^{\frac{3}{4}}$. So, the combination of the IR cut-off with the entropy gives the energy density of the HDE model. The standard HDE model depends on Bekenstein-Hawking entropy $S=\frac{A}{4 G}$, where $A=4 \pi L^{2}$, thus the density is $\rho_{D}=\frac{3 c^{2}}{8 \pi G} L^{-2}$, where $c$ is numerical constant. It must be stressed that this expression of $\rho_{D}$ is obtained by combining the holographic principle and the dimensional analysis, instead of adding a DE term into the Lagrangian. Because of this extraordinary characteristic, HDE amazingly contrasts from some other theory of dark energy. The vacuum energy is associated with the UV cut-off and Ricci scalar, particle horizon, Hubble horizon, event horizon, etc. i.e. large scale structure of the Universe, is associated with the infrared (IR) cut-off. The HDE model endures the decision of IR cut-off problem. Numerous investigations of different (IR) cut-off's have been done in Refs. 46, 52,56].

Various entropies are used for the investigation of the cosmological and gravitational incidence. The Tsallis HDE [57], Rényi HDE [58] and Sharma-Mittal HDE [59] are in demand and are extensively studied in literature. Differing from the usual HDE model with Bekenstein entropy, such models give a late-time accelerated Universe. Rényi HDE depicts better stability as its own, in a non-interacting Universe [58]. It is stable and Tsallis HDE [60] is never stable if the Sharma-Mittal HDE becomes dominant in the Universe. So the inferences shows that Rényi and Tsallis entropies can be obtained by Sharma-Mittal entropy 61-63. By considering the Hubble horizon as the IR cut-off, Tsallis HDE in Brans-Dicke cosmology has been studied [64], which demonstrates that both non-interacting and interacting cases are classically unstable. Recently Tsallis agegraphic dark energy model along with pressure-less dust was examined by Zadeh et al. 65] and they observed that these models are classically unstable and show a latetime acceleration in non-interacting cases. Investigation of Sharma-Mittal, Rényi and Tsallis HDE, models have been done in [66] by taking Loop Quantum Cosmology in consideration.

It is important to mention that observations admit an interaction between the dark sectors (DM and DE) of cosmos which can solve the coincidence problem and the tension in current observational values of the Hubble parameter $67-71$. 
HDE models generate late-time acceleration using infrared cut-off with the Hubble horizon when there is some interaction between dark energy and dark matter [27, 72, 77]. It can give late-time acceleration with matter-dominated decelerated expansion in the past. Wei and Cai [78], explored the interacting agegraphic dark energy (IADE) model considering the three most popular forms ( $Q=3 \gamma H \rho_{\text {tot }}, 3 \beta H \rho_{m}, 3 \alpha H \rho_{q}$ [67, 79, 80, where $\gamma, \beta, \alpha$ are constants) of interaction. The cosmological consequences of the recently proposed Tsallis HDE has been investigated by taking the interaction between $\mathrm{DE}$ and dark matter as, $Q=H\left(\alpha \rho_{m}+\beta \rho_{D}\right)$ with IR cutoff as Hubble horizon [81]. Also, considering various IR cut-off, the authors [82], explored the THDE model evolution and investigated their cosmological consequences with an interaction ( $Q=3 b^{2} H\left(\rho_{m}+\rho_{D}\right)$, where $b^{2}$ is coupling constant) between the dark matter and DE of the Universe with Hubble horizon as IR cut-off. Recently, The interaction rate between dark matter and DE has been reconstructed for the HDE models by considering Hubble horizon as IR cut-off with three distinct forms of the interaction term $Q$ 83].

The scenario of interaction between DM and DE is one of such alternative models, which is the subject interest of the present work. In this work, we investigate the evolution of our Universe by considering an interaction between the RHDE and DM whose IR cut-off is the Hubble horizon. The behaviour of the RHDE deceleration parameter, the equation of state parameter EoS, the density parameter has been studied for the present model. Furthermore, we also investigate the stability of the RHDE model in the present scenario. However, the present work has some similarities and differences with other models reported by [78, 81, 83] in different ways. Firstly, in this paper, the functional form of the interaction term is different from 68, 81, 82], while similar to the interaction proposed in [83]. Secondly, this work comprises of the recently proposed RHDE model from three different parametrizations of the interaction term $Q$, while in 83, the HDE model is considered.

The interaction function $\mathrm{Q}$ is supposed to be proportional to $\mathrm{H} \rho_{D}$, where $\mathrm{H}$ is the Hubble parameter and $\rho_{D}$ is the Rényi HDE density. The strength of the interaction depends on the proportionality parameter $\alpha$. Praseetha and Mathew checked at the apparent and event horizon in interacting holographic models whether the second law of thermodynamics is valid [84].

These works are behind our motivation for investigating the cosmological consequence of Rényi HDE model by using infrared cut-off with the Hubble horizon and also by taking three different parametrizations of the interaction function Q, in the context of interacting flat FRW Universe. The organization of the paper is as follows: In Sect. 2, we discuss field equations in flat FRW Universe, the RHDE Model and calculated some cosmological parameters in the interacting RHDE model. In Sect. 3, we analysed the cosmological behaviour of the interacting RHDE for the model I, model II and model II. Finally, in the last section, we concluded outcomes.

\section{Parametrization on the interaction term and RHDE}

The metric for an isotropic and homogeneous flat FRW Universe is given by :

$$
d s^{2}=-d t^{2}+a^{2}(t)\left(d r^{2}+r^{2} d \Omega^{2}\right),
$$


where $\mathrm{a}(\mathrm{t})$ is known as the scale factor. The Hubble parameter is determined as, $H=\frac{\dot{a}}{a}$, where the dot represents derivative with respect to cosmic time. The Friedmann equations, in the form of Hubble parameter are given as,

$$
H^{2}=\frac{1}{3}(8 \pi G)\left(\rho_{D}+\rho_{M}\right)
$$

where $\Omega_{D}=\frac{1}{3} 8 \pi G \rho_{D} H^{-2}$ and $\Omega_{m}=\frac{1}{3} 8 \pi G \rho_{m} H^{-2}$ are the energy density parameters of the RHDE and pressure less matter, respectively, expressed as fractions of critical density $\rho_{c}=\frac{3 H^{2}}{8 \pi G}$. Also, $\rho_{m}$ and $\rho_{D}$ denote the energy density of matter and RHDE, respectively, and $\frac{\rho_{m}}{\rho_{D}}=r$ represents the energy density ratio of two dark components 47, 85. Now Eq. (2) can be written as:

$$
1=\Omega_{D}+\Omega_{m}
$$

The conservation law to the interacting RHDE and matter are found as :

$$
\begin{gathered}
\dot{\rho}_{m}+3 H \rho_{m}=Q, \\
\dot{\rho}_{D}+3 H\left(\rho_{D}+p_{D}\right)=-Q,
\end{gathered}
$$

Here $\mathrm{Q}$ denotes the interaction function and $\omega_{D}=p_{D} / \rho_{D}$ gives the equation of state. Equations 4 and 5 become decoupled for $Q=0$ permitting the autonomous conservation of dark matter and dark energy. The mutual interaction implies a decaying of the holographic energy component into CDM [86 90]. Kim et al. [86], proposed that if there exists a source/sink in the righthand side of the continuity equation, one must be careful to define the EoS. In this case the effective EoS is the only candidate to represent the state of the mixture of two components arising from decaying of the holographic energy into CDM. This is quite different from the noninteracting case. However, it was suggested that one have to use $\omega_{D}^{e f f}$ when considering the interaction [86]. In this study, we have taken three different parametrizations of the interaction function $\mathrm{Q}$. The common form of the interaction function is taken to be $Q=3 \alpha(z) H \rho_{D}$, where $\alpha$ represents the coupling term which is a function of redshift $z$. The rate of interaction between dark matter and dark energy is obtained as $\Gamma=\frac{Q}{\rho_{H}}, 86,91$ and thus it can be expressed as

$$
\Gamma=3 H(z) \alpha(z)
$$

The rate of energy transfer and also the direction of energy flow depend on this term. The dark energy equation of state parameter $\omega_{D}$ is related to the effective equation of state parameter as 86

$$
\omega_{e f f}=\Omega_{D} \omega_{D}
$$

Now the coincidence parameter $(r)$ is defined as $r=\rho_{m} / \rho_{D}$, which is constant in case of HDE in a spatially flat Universe with Hubble horizon as the IR cut-off [92]. The behaviour of this ratio is crucial for the "conventional" form of the "coincidence problem", namely: "why 
are the dark energy and matter densities of precisely the same order today"? In principle, dark energy and matter redshift at different rates [93]. In [93], it is concluded that there exists a preferred class of DE models for which the dynamics of the energy density ratio is entirely determined by the spatial curvature. For vanishing curvature, the energy density ratio remains constant. This feature is highlighted in a broader context and demonstrate that a constant or slowly varying (as the consequence of a non-vanishing spatial curvature) energy density ratio is compatible with a transition from decelerated to accelerated expansion under the condition of a growing interaction parameter. These models are singled out by a dependence $\rho_{D} \propto H^{2}$ where $\rho_{D}$ is the dark energy density. Exactly this dependence is characteristic for a certain type of dark energy models, inspired by the holographic principle [43]. Also, a different way to understand the dynamics of the ratio $r$ can be seen in details 93 .

In this study to reconstruct the interaction function $\mathrm{Q}$, we have taken three different ansatzes which are given in 83 as

Model (I)

$$
\alpha(z)=\alpha_{1}+\alpha_{2}(1+z)
$$

Model (II)

$$
\alpha(z)=\alpha_{1}+\alpha_{2}\left(\frac{z}{1+z}\right)
$$

Model (III)

$$
\alpha(z)=\alpha_{1}+\alpha_{2}\left(\frac{1}{1+z}\right),
$$

Where $\alpha_{1}$ and $\alpha_{2}$ are constant parameters. The model I, II and III have a linear, mixed and inverse dependence on $z$. So, the aforementioned three models lead us to a non-interacting case after reduction, if $\alpha_{1}$ and $\alpha_{2}$ are taken as zero. Here we find two parameters $\alpha_{1}$ and $\alpha_{2}$ since for these three models $r$ is varying. In [83], the distance modulus measurements of type Ia supernova from the Joint Light-curve Analysis (JLA) 94 and the observational measurements of Hubble parameter (OHD) have been used to constrain these model parameters for holographic dark energy models. Cosmic Chronometer method [95], measurements from galaxy distribution [96] and from Lymann $-\alpha$ forest distribution 97 methods are used to measure the OHD. In this work, we use different values of $\alpha_{1}$ and $\alpha_{2}$ to see the models behaviour since the interaction function $Q$ depends on the parameters $\alpha_{1}$ and $\alpha_{2}$. A negative $Q$ shows the flow of energy from the dark matter to the dark energy and a positive $Q$ shows the reverse.

The form of the Bekenstein entropy of a system is $S=\frac{A}{4}$, where $A=4 \pi L^{2}$ and $\mathrm{L}$ is the IR cut-off. Another modified form of the Rényi entropy [58] is given as:

$$
S=\frac{1}{\delta} \log \left(\frac{\delta}{4} A+1\right)=S=\frac{1}{\delta} \log \left(\pi \delta L^{2}+1\right)
$$

Rényi HDE density, by considering the assumption $\rho_{d} d V \propto T d S$, takes the following form: 

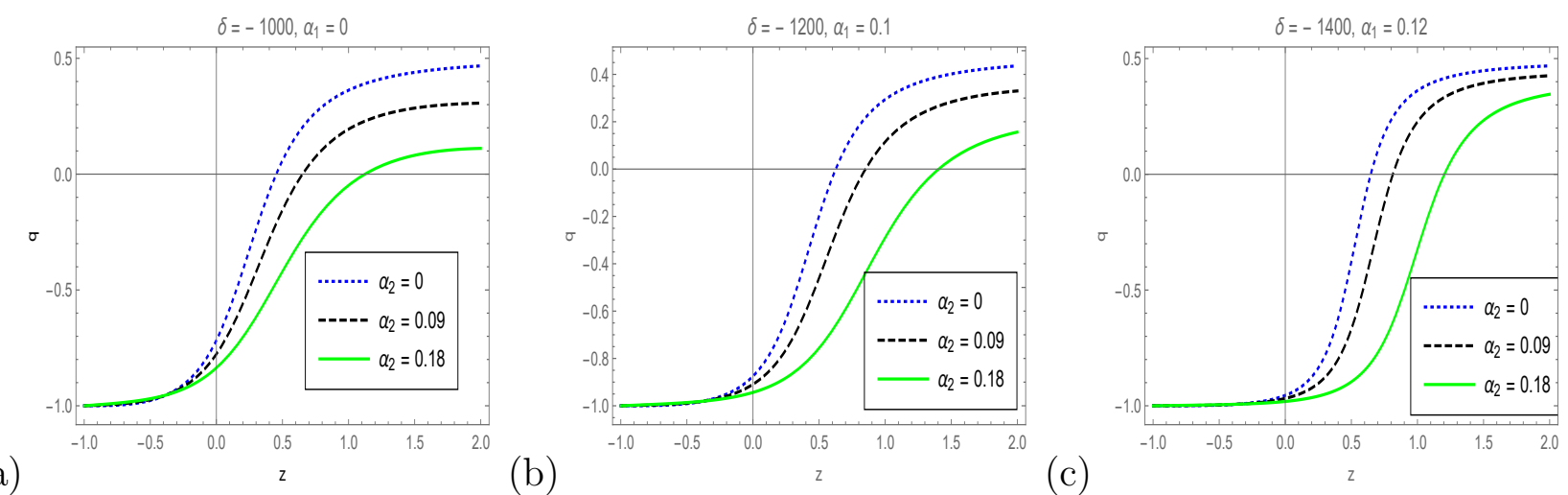

Figure 1: The evolution of deceleration parameter $(q)$ in the RHDE model (I) versus redshift $z$ for different values of model parameter $\delta, \alpha_{1}$, and $\alpha_{2}$ where $H_{0}=69.6, \Omega_{D 0}=0.70$.

$$
\rho_{D}=\frac{3 c^{2}}{8 \pi L^{2}}\left(\pi \delta L^{2}+1\right)^{-1}
$$

By taking Hubble horizon as an IR cut-off $L=\frac{1}{H}$, we obtained:

$$
\rho_{D}=\frac{3 c^{2} H^{2}}{8 \pi\left(\frac{\pi \delta}{H^{2}}+1\right)}
$$

where $c^{2}$ is a numerical constant as usual. Now, by the definition of $r$, we obtain 86

$$
\begin{gathered}
r=\frac{\rho_{m}}{\rho_{D}}=\frac{\frac{1}{3} \rho_{m} M_{p}^{-2} H^{-2}}{\frac{1}{3} \rho_{D} M_{p}^{-2} H^{-2}}=\frac{\Omega_{m}}{\Omega_{D}}=\frac{1-\Omega_{D}}{\Omega_{D}} \\
r=\frac{1}{\Omega_{D}}-1 .
\end{gathered}
$$

For the flat Universe, we have $1-\Omega_{D}=\Omega_{m}$. Moreover, in this case $r$ stays varying. Now, taking the time derivative of Eq. (13), we get

$$
\dot{\rho_{D}}=2 \frac{\dot{H}}{H} \rho_{D}\left(\frac{\pi \delta}{\pi \delta+H^{2}}+1\right),
$$

by taking the time derivative of Eq. (2) and using the Eq. (4) and Eq. (16), and combining the result with Eq. (3), we obtain

$$
\frac{\dot{H}}{H^{2}}=-\frac{3}{2}\left(1+\Omega_{D} \omega_{D}\right)
$$

Now using Eq. (17) we get deceleration parameter $q$

$$
q=-1-\frac{\dot{H}}{H^{2}}=\frac{1}{2}\left(3 \omega_{D} \Omega_{D}+1\right)
$$


Now substituting Eq. (16) in Eq.(5). We get EoS parameter as:

$$
\omega_{D}=-1-\alpha-\frac{2 \pi \delta \dot{H}}{3 H^{2}\left(\pi \delta+H^{2}\right)}-\frac{2 \dot{H}}{3 H^{2}},
$$

Taking time derivative of $\Omega_{D}=\frac{8 \pi G \rho_{D}}{3 H^{2}}$ and by using Eq. 16 , we get

$$
\dot{\Omega_{D}}=\frac{2 \pi \delta \dot{H} \Omega_{D}}{H\left(\pi \delta+H^{2}\right)},
$$

Finally, we explore the stability of the RHDE model as:

$$
v_{s}^{2}=\frac{d p_{D}}{d \rho_{D}}=\frac{\rho_{D}}{\dot{\rho}_{D}} \dot{\omega}_{D}+\omega_{D}
$$

Physical values of the dark energy squared sound speed should lie in the region $0 \leq v_{s}^{2} \leq 1$. Outside of this region, one finds gradient / tachyonic instabilities and/or instabilities connected to superluminal propagation. In the standard scenario, one fixes $v_{s}^{2}=1$. Hence, it is required to check what is the impact of reducing the dark energy squared sound speed to values as low as 0 , and whether the effects on the CMB can be mimicked by dark energy baryon scattering 98 . One important point to note is that to study the effect of the DE squared sound speed, one must again consider values of the DE $\operatorname{EoS} \omega_{D} \neq-1$ since a cosmological constant has no perturbations and hence a cosmology with DE in the form of a cosmological constant has no sensitivity to the DE sound speed. The impact of the DE sound speed on the CMB power spectrum has been discussed in detail in $[99]$. There it was found that the effect of decreasing $v_{s}^{2}$ from 1 to 0 is to suppress the late-time integrated Sachs-Wolfe (LISW) effect when the DE EoS satisfies $\omega_{D}>-1$, but for $\omega_{D}<-1$, decreasing the sound speed results in an enhancement of the LISW effect. Heuristically, at least for the case where $\omega_{D}>-1$, this can be understood as follows: the more we decrease $v_{s}^{2}$, the more DE can cluster and effectively behave as "cold" dark energy. Clustering enhances the DE perturbations, which protect the potentials from decaying, thus leading to a smaller contribution to the late-time integrated Sachs-Wolfe (LISW) effect. We refer the reader to $[99]$ for a mathematically rigorous discussion of the effect of $v_{s}^{2}$ on the CMB. See also e.g. 100 106] for further works examining the effect of $v_{s}^{2}$ on cosmological observations.

\section{Cosmological behaviour of the interacting RHDE}

In this section, we investigate the cosmological behaviour of the deceleration parameter $(q)$, equation of state parameter $\operatorname{EoS}\left(\omega_{D}\right)$, total energy density parameter $\left(\Omega_{D}\right)$ and squared sound speed $\left(v_{s}^{2}\right)$ via three ansatzes mentioned above for the parametrization of interaction term for different values of the Rényi parameter $\delta$. In the following subsections we consider each model, in turn and elaborate parameters numerically, imposing the initial conditions $H(z=0)=69.6$, $\Omega_{D}(z=0)=0.70$.

\subsection{Model I}

For analysis of the RHDE models, we have taken different values of the parameters $\alpha_{1}, \alpha_{2}$, and $\delta$. Figs. 1(a), 1(b) and 1(c), depict the behaviour of the deceleration parameter $q$ versus 

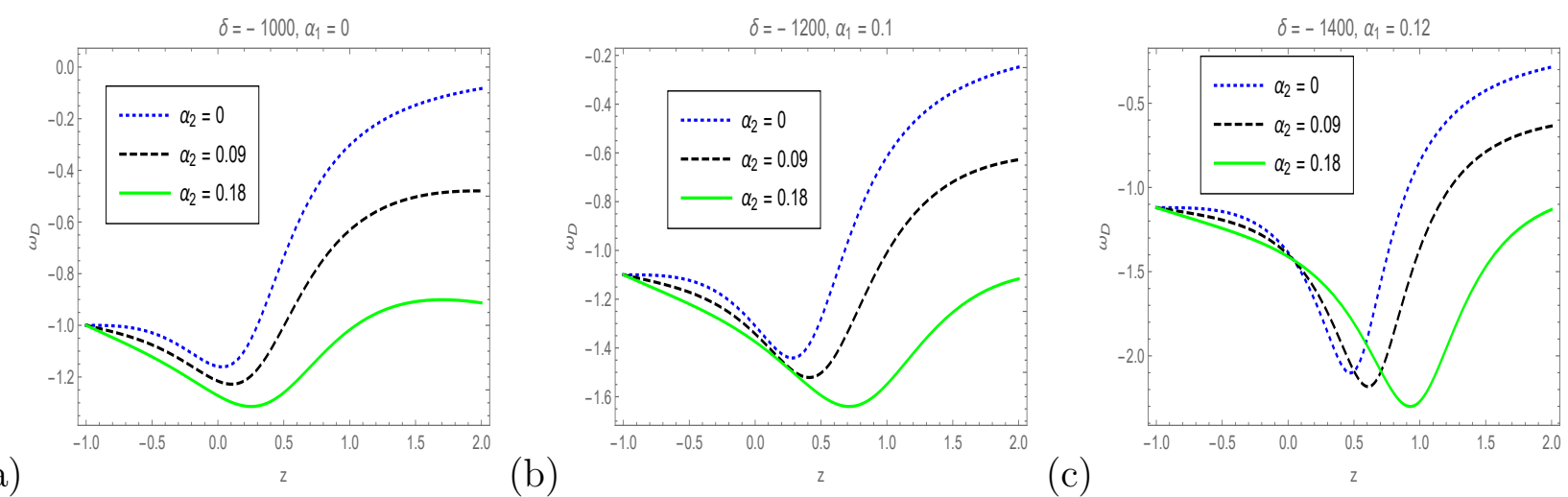

Figure 2: The evolution of EoS parameter $\omega_{D}$ in RHDE model (I) versus redshift $z$ for different values of model parameter $\delta, \alpha_{1}$, and $\alpha_{2}$ where $H_{0}=69.6, \Omega_{D 0}=0.70$.

(a)

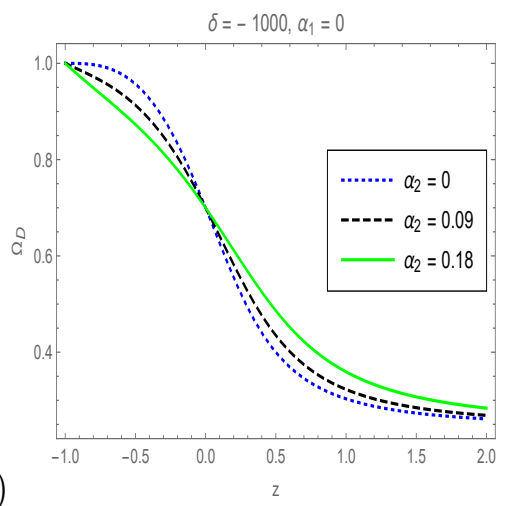

(b)

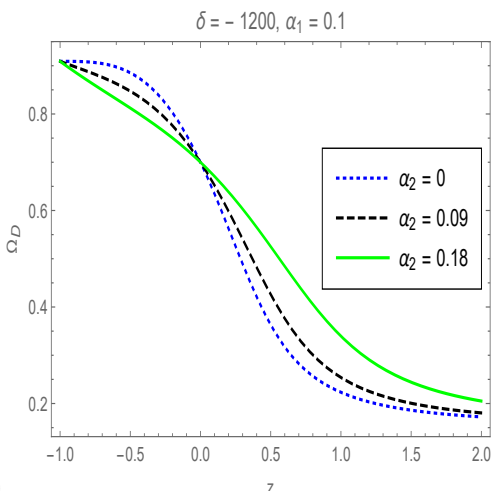

(c)

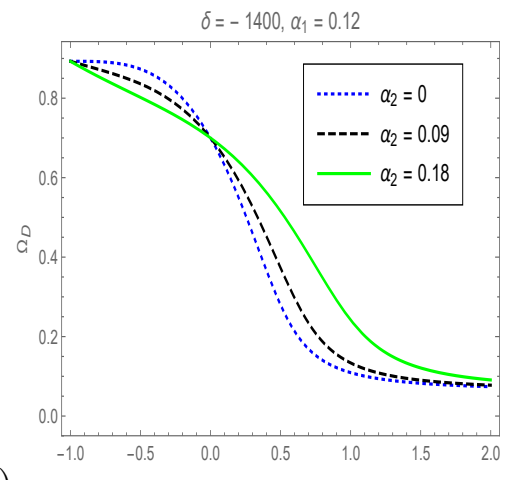

Figure 3: The evolution of energy density parameter $\Omega_{D}$ in RHDE model (I) versus redshift $z$ for different values of model parameter $\delta, \alpha_{1}$, and $\alpha_{2}$ where $H_{0}=69.6, \Omega_{D 0}=0.70$.

(a)

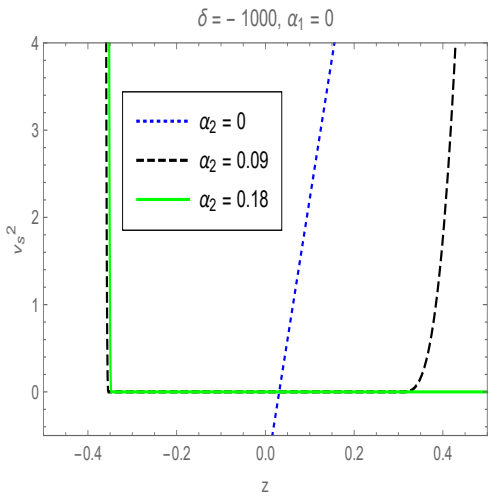

(b)
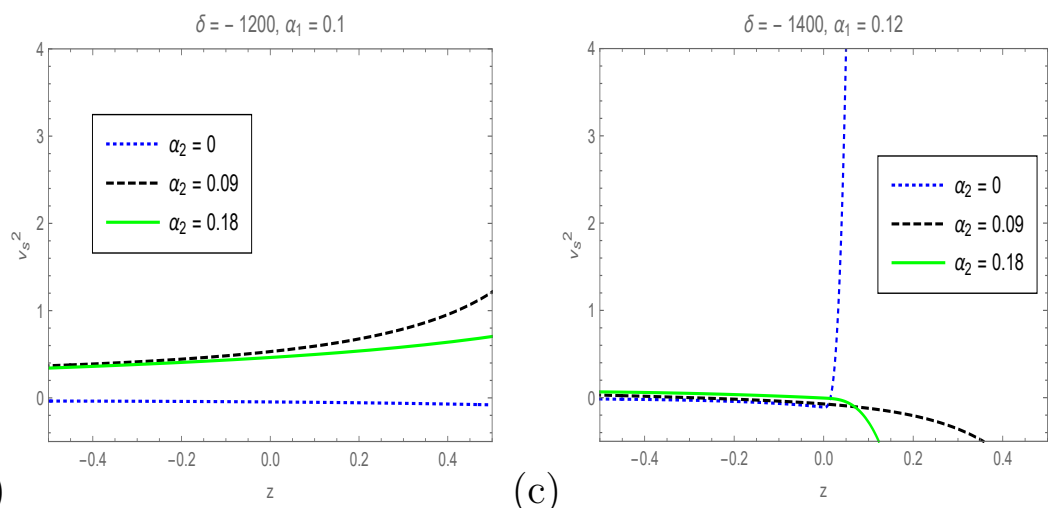

(c)

Figure 4: The evolution of square of the sound speed parameter $v_{s}^{2}$ in RHDE model (I) versus redshift $z$ for different values of model parameter $\delta, \alpha_{1}$, and $\alpha_{2}$ where $H_{0}=69.6, \Omega_{D 0}=0.70$. 

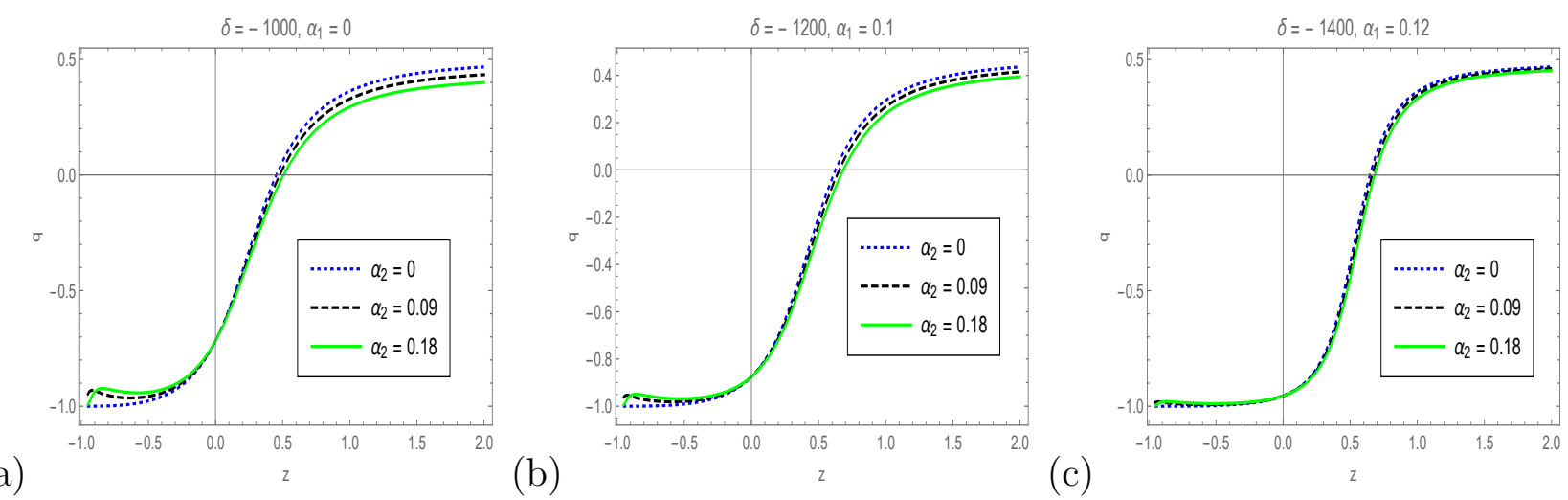

Figure 5: The evolution of deceleration parameter $(q)$ in the RHDE model (II) versus redshift $z$ for different values of model parameter $\delta, \alpha_{1}$, and $\alpha_{2}$ where $H_{0}=69.6, \Omega_{D 0}=0.70$.

(a)

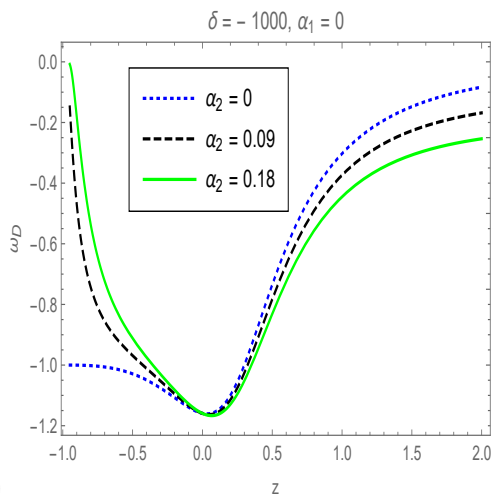

(b)

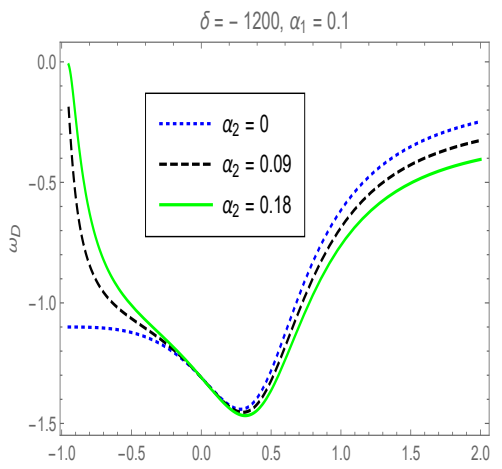

(c)

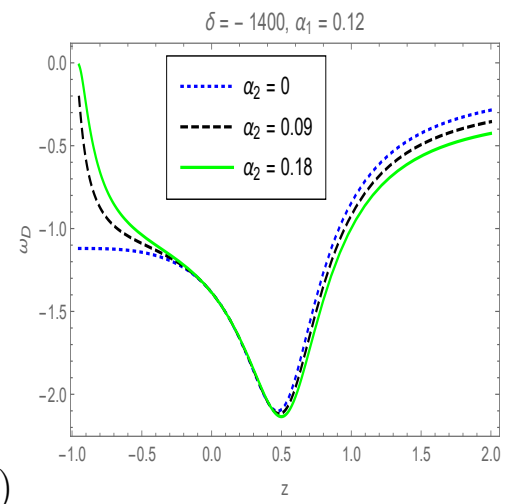

Figure 6: The evolution of EoS parameter $\omega_{D}$ in RHDE model (II) versus redshift $z$ for different values of model parameter $\delta, \alpha_{1}$, and $\alpha_{2}$ where $H_{0}=69.6, \Omega_{D 0}=0.70$.

(a)

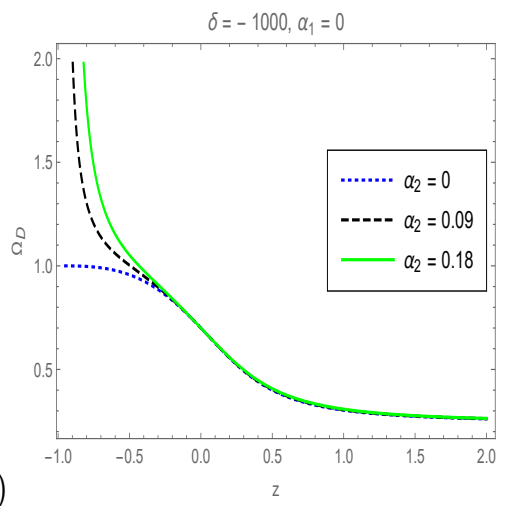

(b)

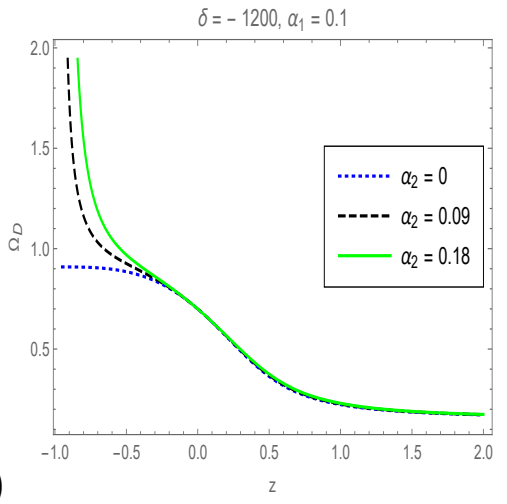

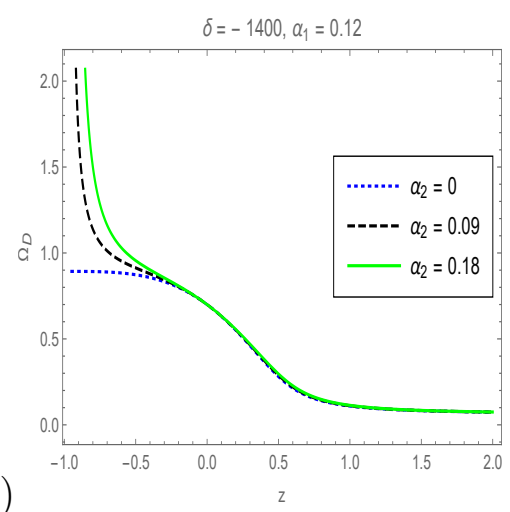

(c)
Figure 7: The evolution of energy density parameter $\Omega_{D}$ in RHDE model (II) versus redshift $z$ for different values of model parameter $\delta, \alpha_{1}$, and $\alpha_{2}$ where $H_{0}=69.6, \Omega_{D 0}=0.70$. 
(a)

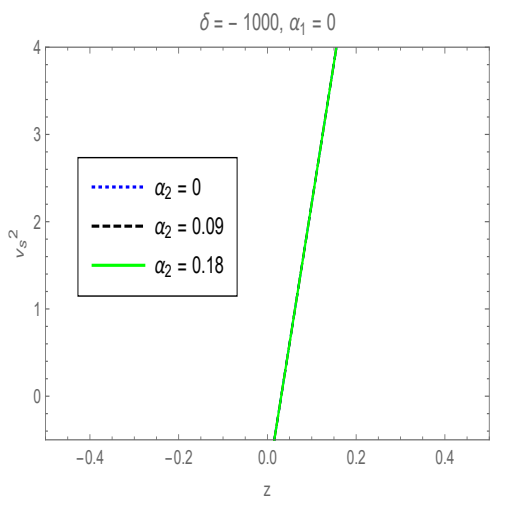

(b)

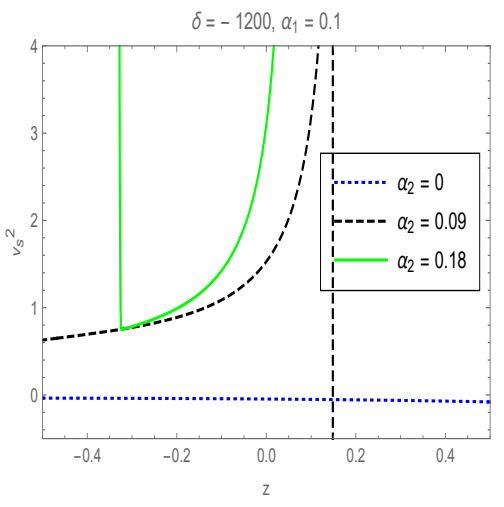

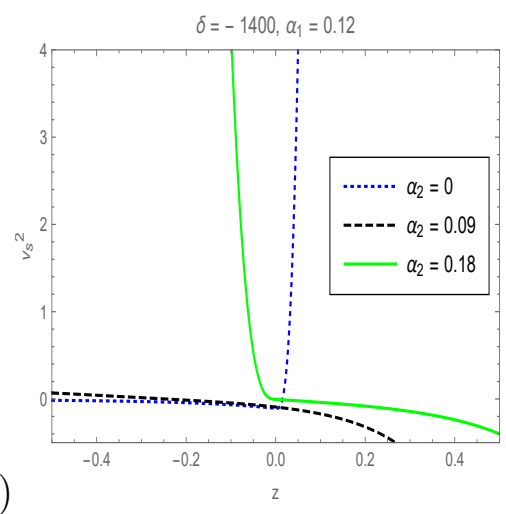

(c)

Figure 8: The evolution of square of the sound speed parameter $v_{s}^{2}$ in RHDE model (II) versus redshift $z$ for different values of model parameter $\delta, \alpha_{1}$, and $\alpha_{2}$ where $H_{0}=69.6, \Omega_{D 0}=0.70$.

(a)
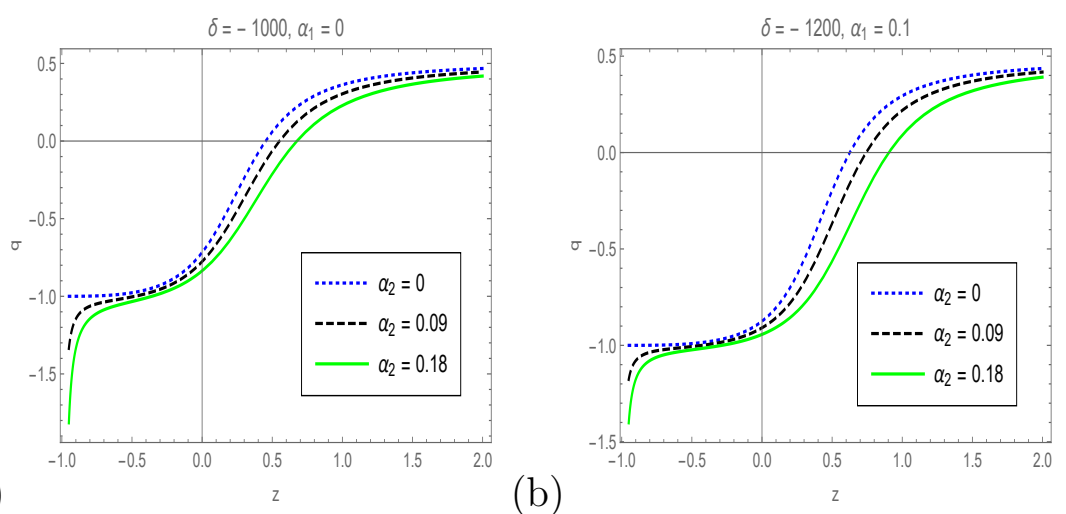

(c)

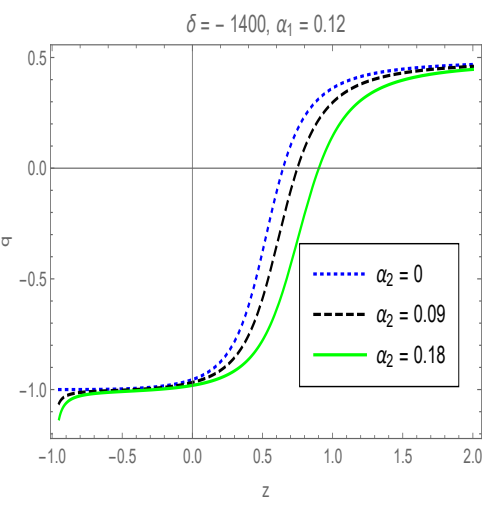

Figure 9: The evolution of deceleration parameter $(q)$ in the RHDE model (III) versus redshift $z$ for different values of model parameter $\delta, \alpha_{1}$, and $\alpha_{2}$ where $H_{0}=69.6, \Omega_{D 0}=0.70$. 
redshift $z$ for different values of $\alpha_{1}, \alpha_{2}$, and $\delta$. In Fig. 1(a), we fix the parameters $\alpha_{1}=0$, and $\delta=-1000$, while we vary $\alpha_{2}$. It is important to mention that the scenario, $\alpha_{1}=0, \alpha_{2}=0$ in this figure and all remaining figures corresponds to the non-interacting case. And, in Figs. $1(\mathrm{~b})$ and 1(c), we vary $\alpha_{1}$, and $\delta$ for the different values of $\alpha_{2}$ as in Fig. 1(a). We observe from Fig. 1, that $q$ changes its sign from positive to negative. Hence model I shows a transition from early decelerated phase to present accelerating phase of the Universe for different values of $\alpha_{1}, \alpha_{2}$ and $\delta$. Fig. 2 shows the evolution of the EoS parameter $\omega_{D}$ versus redshift $z$ for the different choices of $\alpha_{1}, \alpha_{2}$, and $\delta$. From Fig. 2(a), we observe that EoS parameter $\omega_{D}$ varies from quintessence to the phantom era $\omega_{D}<-1$ for $\left(\alpha_{1}=0, \alpha_{2}=0\right),\left(\alpha_{1}=0, \alpha_{2}=0.09\right)$ and $\left(\alpha_{1}=0, \alpha_{2}=0.18\right)$. Finally, converges to the cosmological constant $\omega_{D}=-1$ at future for all the choices of $\alpha_{1}, \alpha_{2}$. The behaviour for the EoS parameter in Figs. 2(b) and 2(c) for the different choices of $\alpha_{1}, \alpha_{2}$, and $\delta$ is noticed, which is different from Fig. 2(a) for $\alpha_{2}=0.18$. It is also observed from Figs. 2(b) and 2(c), the EoS parameter remains in the phantom era throughout the evolution for $\alpha_{2}=0.18$. This indicates that for the interacting RHDE model, $\alpha$ has the linear dependence on $z$. In particular, in [107] it was shown that an interaction is mildly favoured by the combined analysis of several observational data and the equation of state $\omega_{D}$ could be of phantom character, that means $\omega_{D}<-1$.

Fig. 3(a), 3(b) and 3(c) describe the behaviour of dark energy density parameter $\Omega_{D}$ with redshift $z$ for the different choices of $\alpha_{1}, \alpha_{2}$, and $\delta$. We observe that the Universe is dominated by dark energy at present and also in future. It can also be observed from Fig. 3, the effect of interaction increases as we increase the value of $\alpha_{1}$ and decrease the value of $\delta$.

Now, we discuss the stability of the RHDE model. For this purpose, an important quantity is the squared speed of sound $v_{s}^{2}$ as we mentioned before. The $v_{s}^{2} \geq 0$ (real value of speed), shows a regular propagating mode for a density perturbation. For $v_{s}^{2}<0$, the perturbation becomes an irregular wave equation. Hence the negative squared speed (imaginary value of speed) shows an exponentially growing mode for a density perturbation. That is, an increasing density perturbation induces a lowering pressure, supporting the emergence of instability [108 110]. The squared speed of the sound $v_{s}^{2}$ of the model I has also been characterized for the stability of the RHDE model. It is plotted in Fig. 4 versus redshift $z$ for the different values of $\alpha_{1}, \alpha_{2}$, and $\delta$.

From Fig. 4(a), we observe that the RHDE model is not stable for $\alpha_{1}=0$ and $\alpha_{2}=0$ since the velocity becomes imaginary. While the RHDE model is stable between $-0.3<z<0.3$ for $\alpha_{1}=0, \alpha_{2}=0.09$ and $\alpha_{2}=0.18$. The velocity becomes imaginary at $z=-0.3$ and $z=0.3$ for $\alpha_{1}=0, \alpha_{2}=0.09$ and $\alpha_{2}=0.18$, at both points, the RHDE model is unstable. Now from Fig. 4 (b), it is clear that the RHDE model is stable for all values of $z$ for $\alpha_{2}=0.09$ and $\alpha_{2}=0.18$, but for $\alpha_{2}=0$ the RHDE model is stable only at a late time. Now Fig. 4(c) shows that the RHDE model is stable for all values of $\alpha_{1}$ and $\alpha_{2}$ in the region $z<0$. While in the region $z>0$ the velocity becomes imaginary for all values of $\alpha_{1}$ and $\alpha_{2}$. We can also observe from Fig. 4(a), the squared sound speed $v_{s}^{2}$ changes suddenly from close to being 0 to 4 at $z=-0.35$, and similarly around $z=0$ in Fig. 4(c). The net effect of increasing $v_{s}^{2}$ is higher ISW (Integrated Sachs-Wolfe) power. This reflects the increased potential decay due to dark energy; while dark energy perturbations would help preserve the potential, increasing $v_{s}^{2}$ reduces the dark energy perturbation contribution and so eases the decay of the potential. The sqaured sound speed $v_{s}^{2}$ is also obtained in terms of the redshift parameter filled with variable modified Chaplygin gas (VMCG) in the FRW Universe [111]. The authors have shown the dependence of the velocity of sound $v_{s}^{2}$ on the free parameter $n$ as a function of redshift $z$ [111]. In [111], it is observed that the velocity has a magnitude lying below unity, and then as the redshift $\mathrm{z}$ became smaller, 
(a)

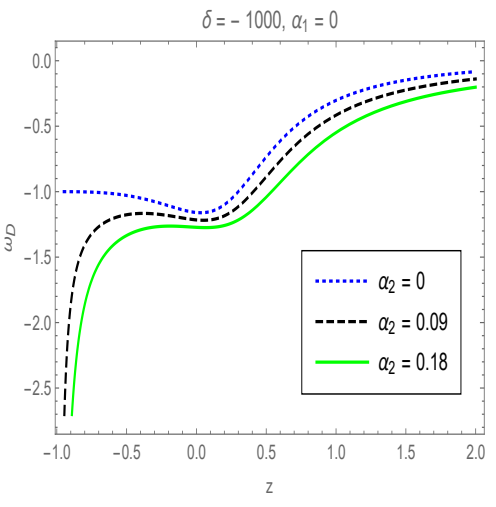

(b)

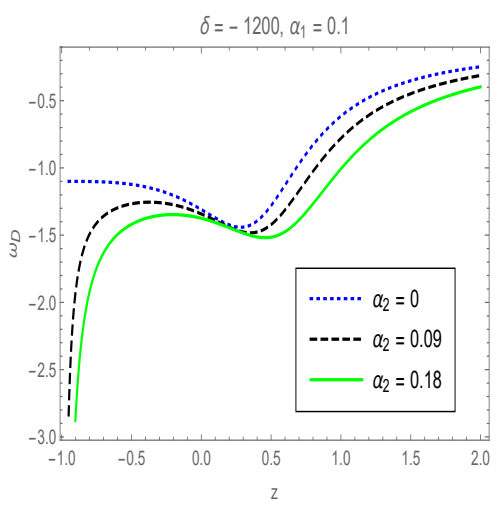

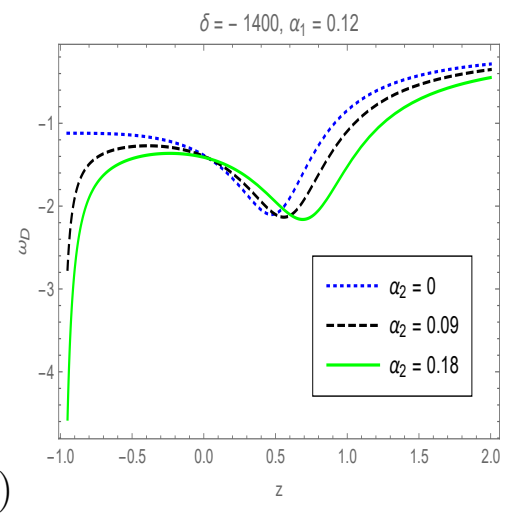

Figure 10: The evolution of EOS parameter $\omega_{D}$ in RHDE model (III) versus redshift $z$ for different values of model parameter $\delta, \alpha_{1}$, and $\alpha_{2}$ where $H_{0}=69.6, \Omega_{D 0}=0.70$.

the velocity of sound increased rapidly for $n<0$ (phantom dominated universe). After that the velocity becomes imaginary. They proposed that for $n<0$ indicates a perturbative cosmology and favors structure formation in the Universe [111, 112].

\subsection{Model II}

In model II, the deceleration parameter $(q)$ is plotted as a function of $z$ in Figs. 5(a), 5(b) and $5(\mathrm{c})$ by considering different values of the parameter $\alpha_{1}, \alpha_{2}$ and $\delta$. It also shows that $q$ goes towards negative from the positive region which depicts the transition of the Universe from early decelerated phase to present accelerating phase for all choices of $\alpha_{1}, \alpha_{2}$ and $\delta$ similar to the Model I.

Figs. 6(a), 6(b) and 6(c) show the evolution of the EoS parameter $\omega_{D}$ versus redshift $z$ for different values of the parameter $\alpha_{1}, \alpha_{2}$ and $\delta$. This depicts that the EoS parameter $\omega_{D}$ varies in both quintessence era $\omega_{D}>-1$ and the phantom era $\omega_{D}<-1$. It is also observed that the Model II lies in quintessence era $\omega_{D}>-1$ in the future for some choices of $\alpha_{1}, \alpha_{2}$ and $\delta$ and in phantom era $\omega<-1$ for $\alpha_{2}=0$ at future. From Figs. 7(a), 7(b) and 7(c), we observe that behaviour of the energy density parameter $\Omega_{D}$ in RHDE model (II) versus redshift $z$ for different values of model parameter $\delta$ in flat FRW Universe for different $\alpha_{1}$, $\alpha_{2}$ is different from model I and increases in low redshift region. In Fig. 7, the energy density parameter $\Omega_{D}$ grows larger than 1 for some choices of $\alpha_{2}$, which seems to be impossible according to the definition of $\Omega_{m}$. This can be understood as, for $\rho_{D}=\frac{3 c^{2} H^{2}}{8 \pi\left(\frac{\pi \delta}{H^{2}}+1\right)}$, the energy density ratio $r$ is variable, which makes the energy density parameter of the RHDE $\Omega_{D}$ time-dependent. Now from $\omega_{\text {eff }}=\Omega_{D} \omega_{D}$ and $\Gamma=3 H(z) \alpha(z)$ relationship, we can say that the increasing value of $\alpha_{2}$ leads the range of $\Omega_{D}$ to widen by reason of advance of the dark matter into dark energy. The similar conclusion has also been drawn in [113, 114]. From Fig. 8(a), we observe that the RHDE model is unstable $v_{s}^{2}<0$ at present for all values of $\alpha_{1}, \alpha_{2}$, and $\delta$. It can be seen that the RHDE model is stable $v_{s}^{2} \geq 0$ in the future for all values of $\alpha_{1}, \alpha_{2}$, and $\delta$ as shown in Figs. $8(\mathrm{~b})$ and $8(\mathrm{c})$. 

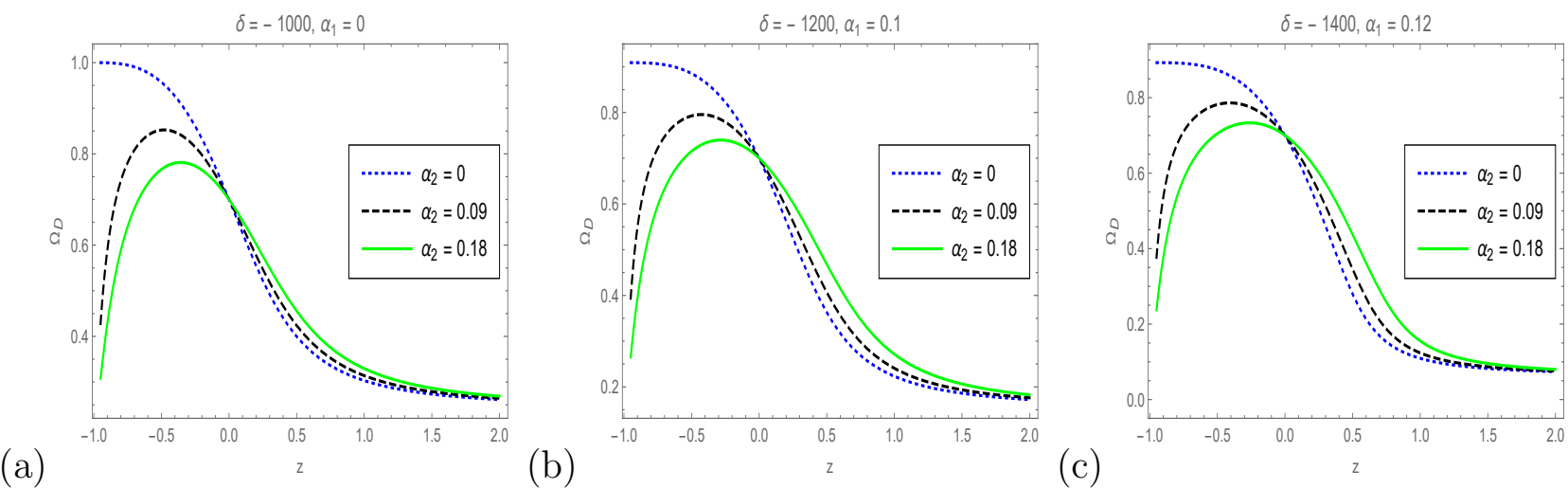

Figure 11: The evolution of energy density parameter $\Omega_{D}$ in RHDE model (III) versus redshift $z$ for different values of model parameter $\delta, \alpha_{1}$, and $\alpha_{2}$ where $H_{0}=69.6, \Omega_{D 0}=0.70$.

(a)

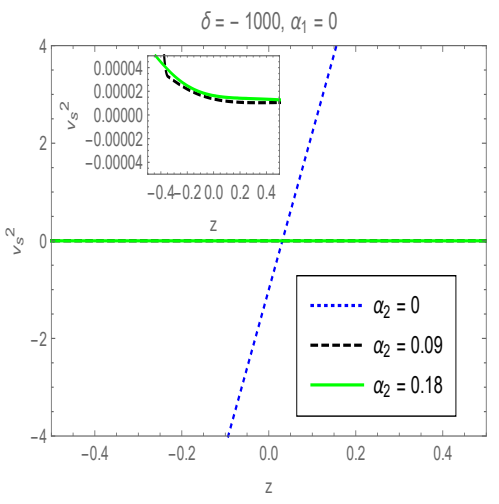

(b)
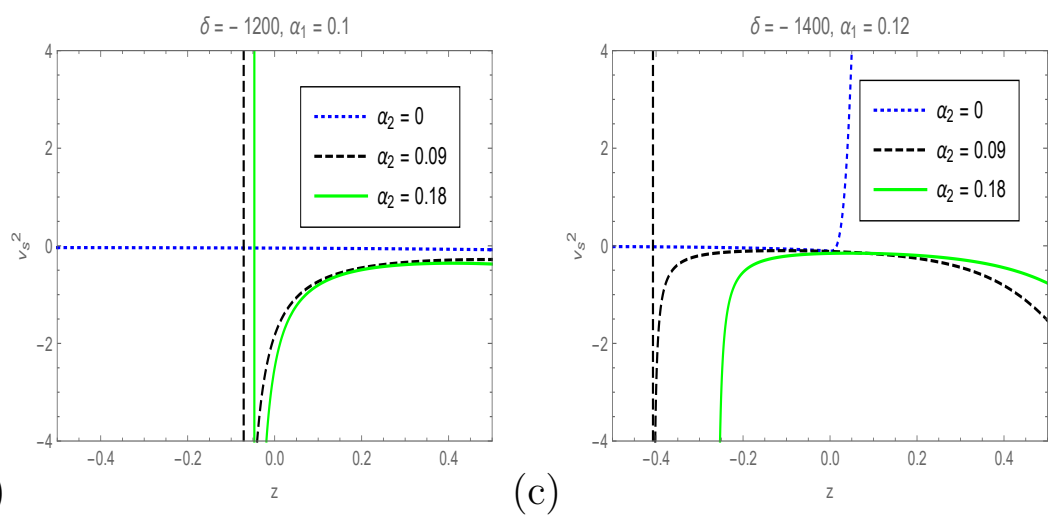

Figure 12: The evolution of square of the sound speed parameter $v_{s}^{2}$ in RHDE model (III) versus redshift $z$ for different values of model parameter $\delta, \alpha_{1}$, and $\alpha_{2}$ where $H_{0}=69.6, \Omega_{D 0}=$ 0.70 . 


\subsection{Model III}

Figs. 9(a), 9(b) and 9(c), depict the behaviour of the deceleration parameter $q$ versus redshift $z$ for different values of $\alpha_{1}, \alpha_{2}$, and $\delta$. We observe from Fig. 9, that $q$ changes its sign from positive to negative for all values of $\alpha_{1}, \alpha_{2}$, and $\delta$. Therefore, model III shows a transition from early decelerated phase to present accelerating phase of the Universe for different values of $\alpha_{1}, \alpha_{2}$, and $\delta$. Fig. 10(a), 10(b) and 10(c), exhibit the evolution of the EoS parameter $\omega_{D}$ versus redshift $z$ for the different choices of $\alpha_{1}, \alpha_{2}$, and $\delta$. From Fig. 10(a), we observe that EoS parameter $\omega_{D}$ varies from quintessence to the phantom era $\omega_{D}<-1$ for for all values of $\alpha_{1}, \alpha_{2}$, and $\delta$, and remains in the phantom era at future except for $\left(\alpha_{1}=0, \alpha_{2}=0\right)$ for $\delta=-1000$. The EoS parameter remains in the phantom era for all choices of $\alpha_{1}, \alpha_{2}$, and $\delta$ at future, which can be observed in Figs. 10(b) and 10(c).

Fig. 11(a), 11(b) and 11(c) show the behaviour of dark energy density parameter $\Omega_{D}$ with redshift $z$ for the different choices of $\alpha_{1}, \alpha_{2}$, and $\delta$. It can also be observed from Fig. 11, that the variation in both $\alpha_{1}$ and $\alpha_{2}$ contributes to the evolutionary behaviour of the RHDE density parameter $\Omega_{D}$. This is particularly noticeable in Fig. 11, the density parameter $\Omega_{D}$ decreases in the future. In this process, the interaction impacts on both the RHDE and the DM. Accordingly, their contents are changing due to the energy transfer from the RHDE to the DM. The squared speed of the sound $v_{s}^{2}$ versus $z$ of the model III has been plotted in Fig. 12. From Fig. 12(a), we observe that the RHDE model is unstable $v_{s}^{2}<0$ in the future without interaction i.e. $\alpha_{1}=0$ and $\alpha_{2}=0$. Also, for $\alpha_{2}=0.09$ and $\alpha_{2}=0.18$, the curves look to be overlapping. The inner plot of Fig. 12(a) shows a close-up of the outer plot in which the difference can be seen. They are not exactly identical but difference is very small. We can also observe from Fig. 12(b), that the RHDE model is unstable $v_{s}^{2} \leq 0$ in the past for $\alpha_{2}=0.09$, $\alpha_{2}=0.18$. And, the model is stable for $\alpha_{2}=0$ as can be seen in Fig. 12(c).

\section{Conclusion}

This work comprises the study of the RHDE model where Hubble horizon is taken as the infrared cut-off by taking three different parametrizations of the interaction term in the context of flat FRW Universe. Different values of the parameters $\alpha_{1}, \alpha_{2}$ and $\delta$ are taken for the interaction of dark matter and dark energy in the derived model. Following are results which we obtained on the basis of the graphical analysis:

* The sign of deceleration parameter $q(z)$ indicates whether the model inflates or not. The deceleration parameter $q$ shows a transition from early decelerated phase to present accelerating phase of the Universe for all choices of the parameters $\alpha_{1}, \alpha_{2}$ and $\delta$ in all three models (I, II, III). The deceleration parameter $q(z)$ shows that it increases with redshift and there is a transition in the signature of $q(z)$ with the variation in $\alpha_{1}, \alpha_{2}$ and $\delta$.

* The RHDE equation of state parameter (EoS) $\omega_{D}$ eventually approaches to values negative enough to generate the accelerated expansion. The present value of $\omega_{D}$ remains in phantom regime $\left(\omega_{D} \leq-1\right)$ for Model I, Model II and Model III, though the parametrizations are different in these models. The EoS parameter of Model I and Model II lies in the phantom region at future, while for Model II remains in quintessence era except $\alpha_{2}=0$. Therefore, the equation of state parameter for all three models of the RHDE model shows the different behaviour in 
the low-redshift region (at future).

* We observe that the dark energy density parameter $\Omega_{D}$ is a monotonically increasing function of $z$. For the non-interacting case of $\alpha_{1}=\alpha_{2}=0$, we find that $\Omega_{D} \rightarrow 1$ as $z$ increases as can be seen for all three models, while for the interaction case of $\alpha_{1}=\alpha_{2} \neq 0, \Omega_{D} \neq 1$ for model I, II and III. The first case is obvious because the Rényi holographic energy with the Hubble horizon dominates in the future. Further, the latter shows that two components become comparable, due to the interaction. Similar results have also been found by Kim et al. 86] for the interacting holographic dark energy model with the future event horizon. We also observe that the Model II is not consistent with the observed evolution scenario of the energy density parameter $\Omega_{D}$ for $\alpha_{2}=0.09$ and $\alpha_{2}=0.18$. On the other hand model I and III are consistent with the observed behaviour of the energy density parameter $\Omega_{D}$.

* The graphical behaviour of the squared speed of sound is used to analyse the stability of model I, model II and model III of RHDE. We have noticed that the Rényi HDE models I, II and III with Hubble horizon as IR cut-off are classically stable $\left(v_{s}^{2} \geq 0\right)$ for some choices of the parameters $\alpha_{1}, \alpha_{2}$ and $\delta$ at present and future. But for some choices of the parameters $\alpha_{1}, \alpha_{2}$ and $\delta$, all three models of the RHDE are unstable $v_{s}^{2}<0$.

In summary, the evolutionary behaviour of the deceleration parameter $(q)$, equation of state parameter EoS $\left(\omega_{D}\right)$, total energy density parameter $\left(\Omega_{D}\right)$ and squared sound speed $\left(v_{s}^{2}\right)$ has been investigated for the different choices of $\alpha_{1}, \alpha_{2}$ and $\delta$. It is observed that the behaviour of the deceleration parameter $(q)$, total energy density parameter $\left(\Omega_{D}\right)$, the EoS parameter $\left(\omega_{D}\right)$ and squared sound speed $\left(v_{s}^{2}\right)$ for all three models are different from high red-shift region to low redshift region for some choices of $\alpha_{1}, \alpha_{2}$ and $\delta$. While, the behaviour of cosmological parameters of model III is similar to the model I and model II for some choices of $\alpha_{1}, \alpha_{2}$ and $\delta$.

For the future work, different observational data sets such as the distance modulus measurements of type Ia supernova from the Joint Light-curve Analysis and the observational measurements of Hubble parameter can be utilized to reconstruct the model parameters to figure out the correct model and to understand the nature of the RHDE.

\section{Acknowledgements}

We are very much grateful to the honourable referee for the precious time and illuminating comments that have significantly improved our work in terms of research quality and presentation.

\section{References}

[1] A. G. Riess et al. [Supernova Search Team], "Observational evidence from supernovae for an accelerating universe and a cosmological constant," Astron. J. 116 (1998) 1009. doi:10.1086/300499. 
[2] S. Perlmutter et al. [Supernova Cosmology Project Collaboration], "Measurements of $\Omega$ and $\Lambda$ from 42 high redshift supernovae," Astrophys. J. 517 (1999) 565. doi:10.1086/307221 astro-ph/9812133|.

[3] P. de Bernardis et al. [Boomerang Collaboration], "A Flat universe from high resolution maps of the cosmic microwave background radiation," Nature 404 (2000) 955 doi: $10.1038 / 35010035$

[4] R. A. Knop et al. [Supernova Cosmology Project Collaboration], "New constraints on Omega(M), Omega(lambda), and w from an independent set of eleven high-redshift supernovae observed with HST," Astrophys. J. 598 (2003) 102 doi:10.1086/378560

[5] M. Colless et al. [2DFGRS Collaboration], "The 2dF Galaxy Redshift Survey: Spectra and redshifts," Mon. Not. Roy. Astron. Soc. 328 (2001) 1039 doi:10.1046/j.13658711.2001.04902.x

[6] M. Tegmark et al. [SDSS Collaboration], "Cosmological parameters from SDSS and WMAP," Phys. Rev. D 69 (2004) 103501 doi:10.1103/PhysRevD.69.103501

[7] S. Cole et al. [2dFGRS Collaboration], "The 2dF Galaxy Redshift Survey: Power-spectrum analysis of the final dataset and cosmological implications," Mon. Not. Roy. Astron. Soc. 362 (2005) 505 doi:10.1111/j.1365-2966.2005.09318.x

[8] V. Springel, C. S. Frenk and S. D. M. White, "The large-scale structure of the Universe," Nature 440 (2006) 1137 doi:10.1038/nature04805

[9] S. Hanany et al., "MAXIMA-1: A Measurement of the cosmic microwave background anisotropy on angular scales of 10 arcminutes to 5 degrees," Astrophys. J. 545 (2000) L5 doi: $10.1086 / 317322$

[10] C. B. Netterfield et al. [Boomerang Collaboration], "A measurement by Boomerang of multiple peaks in the angular power spectrum of the cosmic microwave background," Astrophys. J. 571 (2002) 604 doi:10.1086/340118

[11] D. N. Spergel et al. [WMAP Collaboration], "First year Wilkinson Microwave Anisotropy Probe (WMAP) observations: Determination of cosmological parameters," Astrophys. J. Suppl. 148 (2003) 175 doi:10.1086/377226

[12] S. Nojiri and S. D. Odintsov, "The New form of the equation of state for dark energy fluid and accelerating universe," Phys. Lett. B 639 (2006) 144 doi:10.1016/j.physletb.2006.06.065

[13] K. Bamba, S. Capozziello, S. Nojiri and S. D. Odintsov, "Dark energy cosmology: the equivalent description via different theoretical models and cosmography tests," Astrophys. Space Sci. 342 (2012) 155 doi:10.1007/s10509-012-1181-8

[14] V. Sahni, A. Starobinsky, "The case for a positive cosmological $\Lambda$-term," Int. J. Mod. Phys. D 9(04) (2000) 373-443.

[15] P. J. E. Peebles and B. Ratra, "The Cosmological constant and dark energy," Rev. Mod. Phys. 75 (2003) 559. doi:10.1103/RevModPhys.75.559 
[16] T. Padmanabhan, "Dark energy and gravity," Gen. Rel. Grav. 40 (2008) 529. doi:10.1007/s10714-007-0555-7

[17] W. Chen and Y. S. Wu, "Implications of a cosmological constant varying as R**(-2)," Phys. Rev. D 41 (1990) 695. Erratum: [Phys. Rev. D 45, 4728 doi:10.1103/PhysRevD.41.695

[18] S. M. Carroll, "The Cosmological constant," Living Rev. Rel. 4 (2001) 1. doi:10.12942/lrr2001-1

[19] E. J. Copeland and M. Samiet al., "Dynamics of dark energy," Int. J. Mod. Phys. D 15 (2006) 1753. doi:10.1142/S021827180600942X

[20] S. Weinberg, "The Cosmological Constant Problem," Rev. Mod. Phys. 61 (1989) 1. doi:10.1103/RevModPhys.61.1

[21] T. Chiba, "Tracking K-essence," Phys. Rev. D $66 \quad$ (2002) 063514. doi:10.1103/PhysRevD.66.063514

[22] P. J. E. Peebles and B. Ratra, "Cosmology with a time-variable cosmological'constant," Int. J. Mod. Phys. A 325 (1988) L17-L20.

[23] M. S.Turner, "Making sense of the new cosmology". Int. J. Mod. Phys. A, 17 (2002) 180.

[24] A. Y. Kamenshchik, U. Moschella et al., "An Alternative to quintessence," Phys. Lett. B 511 (2001) 265. doi:10.1016/S0370-2693(01)00571-8

[25] R. R. Caldwell and M. Kamionkowski et al., "Phantom energy and cosmic doomsday," Phys. Rev. Lett. 91 (2003) 071301. doi:10.1103/PhysRevLett.91.071301

[26] A. Sen, "Universality of the tachyon potential," J. High Energy Phys. 1999(12)(2000) 027.

[27] M. Li, "A Model of holographic dark energy," Phys. Lett. B 603 (2004) 1 doi:10.1016/j.physletb.2004.10.014

[28] H. Wei and R. G. Cai, "A New Model of Agegraphic Dark Energy," Phys. Lett. B 660 (2008) 113 doi:10.1016/j.physletb.2007.12.030

[29] A. De Felice and S. Tsujikawa, " $f(R)$ theories," Living Rev. Rel. 13 (2010) 3 doi:10.12942/lrr-2010-3

[30] R. Ferraro and F. Fiorini, "Non trivial frames for $f(T)$ theories of gravity and beyond," Phys. Lett. B 702 (2011) 75 doi:10.1016/j.physletb.2011.06.049

[31] P. Horava, "Quantum Gravity at a Lifshitz Point," Phys. Rev. D 79 (2009) 084008 doi:10.1103/PhysRevD.79.084008

[32] P. Horava, "Membranes at Quantum Criticality," JHEP 0903 (2009) 020 doi:10.1088/1126$6708 / 2009 / 03 / 020$

[33] P. Horava, "Spectral Dimension of the Universe in Quantum Gravity at a Lifshitz Point," Phys. Rev. Lett. 102 (2009) 161301 doi:10.1103/PhysRevLett.102.161301 
[34] S.Maity and P. Rudra, (2018). Gravitational Baryogenesis in Ho $\check{r}$ ava-Lifshitz gravity. arXiv preprint arXiv:1802.00313.

[35] C.H. Brans and R.H. Dicke, "Mach's principle and a relativistic theory of gravitation," Phys. Rev. D 124(3) (1961) 925.

[36] G. Cognola, E. Elizalde, S. Nojiri, S. D. Odintsov and S. Zerbini, "Dark energy in modified Gauss-Bonnet gravity: Late-time acceleration and the hierarchy problem," Phys. Rev. D 73 (2006) 084007 doi:10.1103/PhysRevD.73.084007

[37] T. Harko, F. S. N. Lobo, S. Nojiri and S. D. Odintsov, " $f(R, T)$ gravity," Phys. Rev. D 84 (2011) 024020 doi:10.1103/PhysRevD.84.024020

[38] J. Dutta et al., "Cosmological dynamics of mimetic gravity," JCAP 1802 (2018) 041 doi:10.1088/1475-7516/2018/02/041 arXiv:1711.07290 [gr-qc]].

[39] W. Zimdahl and D. Pavon, L. P. Chimento, "Interacting quintessence," Phys. Lett. B 521 (2001) 133 doi:10.1016/S0370-2693(01)01174-1 [astro-ph/0105479]; M. R. Setare, "The Holographic dark energy in non-flat Brans-Dicke cosmology," Phys. Lett. B 644 (2007) 99 doi:10.1016/j.physletb.2006.11.033

[40] W. Yang et al., "Tale of stable interacting dark energy, observational signatures, and the $H_{0}$ tension," JCAP 1809 (2018) 019 doi:10.1088/1475-7516/2018/09/019 arXiv:1805.08252 [astro-ph.CO]].

[41] E. D. Valentino et al., "Interacting dark energy after the latest Planck, DES, and $H_{0}$ measurements: an excellent solution to the $H_{0}$ and cosmic shear tensions." arXiv:1908.04281 (2019).

[42] S. Nojiri and S. D. Odintsov, "Unifying phantom inflation with late-time acceleration: Scalar phantom-non-phantom transition model and generalized holographic dark energy," Gen. Rel. Grav. 38 (2006) 1285 doi:10.1007/s10714-006-0301-6

[43] L. Susskind, "The World as a hologram," J. Math. Phys. 36 (1995) 6377 doi:10.1063/1.531249

[44] P. Horava and D. Minic, "Probable values of the cosmological constant in a holographic theory," Phys. Rev. Lett. 85 (2000) 1610 doi:10.1103/PhysRevLett.85.1610

[45] S. D. Thomas, "Holography stabilizes the vacuum energy," Phys. Rev. Lett. 89 (2002) 081301. doi:10.1103/PhysRevLett.89.081301

[46] S. D. H. Hsu, "Entropy bounds and dark energy," Phys. Lett. B 594 (2004) 13 doi:10.1016/j.physletb.2004.05.020

[47] B. Wang, E. Abdalla, F. Atrio-Barandela and D. Pavon, "Dark Matter and Dark Energy Interactions: Theoretical Challenges, Cosmological Implications and Observational Signatures," Rept. Prog. Phys. 79 (2016) no.9, 096901 doi:10.1088/0034-4885/79/9/096901

[48] S. Wang, Y. Wang and M. Li, "Holographic Dark Energy," Phys. Rept. 696 (2017) 1 doi:10.1016/j.physrep.2017.06.003 
[49] A. Sheykhi, "Holographic Scalar Fields Models of Dark Energy," Phys. Rev. D 84 (2011) 107302 doi:10.1103/PhysRevD.84.107302

[50] B. Hu and Y. Ling, "Interacting dark energy, holographic principle and coincidence problem," Phys. Rev. D 73 (2006) 123510 doi:10.1103/PhysRevD.73.123510

[51] Y. Z. Ma, Y. Gong and X. Chen, "Features of holographic dark energy under the combined cosmological constraints," Eur. Phys. J. C 60 (2009) 303 doi:10.1140/epjc/s10052-009$0876-7$

[52] H. Wei and S. N. Zhang, "Age Problem in the Holographic Dark Energy Model," Phys. Rev. D 76 (2007) 063003. doi:10.1103/PhysRevD.76.063003

[53] C. Gao and F. Wu et al., "A Holographic Dark Energy Model from Ricci Scalar Curvature," Phys. Rev. D 79 (2009) 043511. doi:10.1103/PhysRevD.79.043511

[54] L. N. Granda and A. Oliveros, "Infrared cut-off proposal for the Holographic density," Phys. Lett. B 669 (2008) 275. doi:10.1016/j.physletb.2008.10.017

[55] L. N. Granda and A. Oliveros, "New infrared cut-off for the holographic scalar fields models of dark energy," Phys. Lett. B 671 (2009) 199. doi:10.1016/j.physletb.2008.12.025

[56] K. Karami and J. Fehri, "New holographic scalar field models of dark energy in non-flat universe," Phys. Lett. B 684 (2010) 61. doi:10.1016/j.physletb.2009.12.060

[57] M. Tavayef, A. Sheykhi, K. Bamba and H. Moradpour, "Tsallis Holographic Dark Energy," Phys. Lett. B 781 (2018) 195 doi:10.1016/j.physletb.2018.04.001; V. C. Dubey, U. K. Sharma and A. Beesham, "Tsallis holographic model of dark energy: Cosmic behavior, statefinder analysis and $\omega_{D}-\omega_{D}^{\prime}$ pair in the non-flat universe," Int. J. Mod. Phys. D 28 (2019) no.15, 1950164.

[58] H. Moradpour, S. A. Moosavi, I. P. Lobo, J. P. Morais Graça, A. Jawad and I. G. Salako, "Thermodynamic approach to holographic dark energy and the Rényi entropy," Eur. Phys. J. C 78 (2018) no.10, 829 doi:10.1140/epjc/s10052-018-6309-8; V. C. Dubey, A. K. Mishra, U. K. Sharma, "Diagnosing the Rényi holographic dark energy model in a flat Universe," Astrophys. Space Sci. 365 (2020) 129; U. K. Sharma and V. C. Dubey, "Rényi holographic dark energy in the Brans-Dicke cosmology," Mod. Phys. Lett. A 35, no.34, (2020) 2050281.

[59] A. Sayahian Jahromi, S. A. Moosavi, H. Moradpour, J. P. Morais Graça, I. P. Lobo, I. G. Salako and A. Jawad, "Generalized entropy formalism and a new holographic dark energy model," Phys. Lett. B 780 (2018) 21 doi:10.1016/j.physletb.2018.02.052; U. K. Sharma, V. C. Dubey, "Exploring the Sharma-Mittal HDE models with different diagnostic tools," Eur. Phys. J. Plus 135, (2020) 391. https://doi.org/10.1140/epjp/s13360-02000411-x

[60] C. Tsallis and L. J. L. Cirto, "Black hole thermodynamical entropy," Eur. Phys. J. C 73 (2013) 2487 doi:10.1140/epjc/s10052-013-2487-6

[61] C. Tsallis, "Possible Generalization of Boltzmann-Gibbs Statistics," J. Statist. Phys. 52 (1988) 479. doi:10.1007/BF01016429 
[62] A. Rényi, in Proceedings of the 4th Berkely Symposium on Mathematics, Statistics and Probability (University California Press, Berkeley, CA, 1961) pp. 547-561.

[63] B.D. Sharma and D.P. Mittal, "New non-additive measures of entropy for discrete probability distributions," J. Math. Sci.10 (1975) 28-40

[64] S. Ghaffari, H. Moradpour, I. P. Lobo, J. P. Morais Graça and V. B. Bezerra, "Tsallis holographic dark energy in the Brans-Dicke cosmology," Eur. Phys. J. C 78 (2018) no.9, 706 doi:10.1140/epjc/s10052-018-6198-x

[65] M. Abdollahi Zadeh, A. Sheykhi and H. Moradpour, "Tsallis Agegraphic Dark Energy Model," Mod. Phys. Lett. A 34 (2019) no.11, 1950086 doi:10.1142/S021773231950086X

[66] A. Jawad, K. Bamba, M. Younas, S. Qummer and S. Rani, "Tsallis, Rényi and SharmaMittal Holographic Dark Energy Models in Loop Quantum Cosmology," Symmetry 10 (2018) no.11, 635. doi:10.3390/sym10110635

[67] L. Amendola, "Coupled quintessence," Phys. Rev. D 62 (2000), 043511 doi:10.1103/PhysRevD.62.043511

[68] A. A. Costa, X. D. Xu, B. Wang, E. G. M. Ferreira and E. Abdalla, "Testing the Interaction between Dark Energy and Dark Matter with Planck Data," Phys. Rev. D 89 (2014) no.10, 103531

[69] S. Wang, Y. Z. Wang, J. J. Geng and X. Zhang, "Effects of time-varying $\beta$ in SNLS3 on constraining interacting dark energy models," Eur. Phys. J. C 74 (2014) no.11, 3148

[70] J. H. He, B. Wang, E. Abdalla and D. Pavon, "The Imprint of the interaction between dark sectors in galaxy clusters," JCAP 12 (2010), 022

[71] L. Feng, H. L. Li, J. F. Zhang and X. Zhang, "Exploring neutrino mass and mass hierarchy in interacting dark energy models," Sci. China Phys. Mech. Astron. 63 (2020) no.2, 220401

[72] W. Fischler and L. Susskind, arXiv: hep-th/9806039.

[73] M. Cataldo, N. Cruz, S. del Campo and S. Lepe, "Holographic principle and the dominant energy condition for Kasner type metrics," Phys. Lett. B 509 (2001) 138 doi:10.1016/S03702693(01)00490-7

[74] B. Guberina, R. Horvat and H. Nikolic, "Generalized holographic dark energy and the IR cutoff problem," Phys. Rev. D 72 (2005) 125011 doi:10.1103/PhysRevD.72.125011

[75] D. Pavon and W. Zimdahl, "Holographic dark energy and cosmic coincidence," Phys. Lett. B 628 (2005) 206 doi:10.1016/j.physletb.2005.08.134

[76] S. Sinha and N. Banerjee, "Density perturbation in an interacting holographic dark energy model," arXiv:1911.06520 [gr-qc]].

[77] B. Nayak, "Interacting Holographic Dark Energy, Present Accelerated Expansion and Black Holes," arXiv:1907.12866 [gr-qc]]. 
[78] H. Wei and R. G. Cai, "Interacting Agegraphic Dark Energy," Eur. Phys. J. C 59 (2009), 99-105

[79] Z. K. Guo, R. G. Cai and Y. Z. Zhang, "Cosmological evolution of interacting phantom energy with dark matter," JCAP 05 (2005), 002; H. Wei and R. G. Cai, "Interacting vectorlike dark energy, the first and second cosmological coincidence problems," Phys. Rev. D 73 (2006), 083002

[80] L. P. Chimento, A. S. Jakubi, D. Pavon and W. Zimdahl, "Interacting quintessence solution to the coincidence problem," Phys. Rev. D 67 (2003), 083513

[81] Q. Huang, H. Huang, J. Chen, L. Zhang and F. Tu, "Stability analysis of a Tsallis holographic dark energy model," Class. Quant. Grav. 36 (2019) no.17, 175001

[82] M. A. Zadeh, A. Sheykhi, H. Moradpour and K. Bamba, "Note on Tsallis holographic dark energy," Eur. Phys. J. C 78 (2018) no.11, 940 ; A. A, Mamon, A. H. Ziaie and K. Bamba, "A Generalized Interacting Tsallis Holographic Dark Energy Model and its thermodynamic implications," arXiv preprint arXiv:2004.01593

[83] P. Mukherjee, A. Mukherjee, H. K. Jassal, A. Dasgupta and N. Banerjee, "Holographic dark energy: constraints on the interaction from diverse observational data sets," Eur. Phys. J. Plus 134 (2019) no.4, 147 doi:10.1140/epjp/i2019-12504-7 arXiv:1710.02417 [astroph.CO]].

[84] P. Praseetha and T. K. Mathew, "Evolution of holographic dark energy with interaction term $\boldsymbol{Q} \propto \boldsymbol{H} \boldsymbol{\rho}_{\mathrm{de}}$ and generalized second law," Pramana 86 (2016) no.3, 701. doi:10.1007/s12043-015-1029-1

[85] M. Abdollahi Zadeh, A. Sheykhi and H. Moradpour, "Thermal stability of Tsallis holographic dark energy in nonflat universe," Gen. Rel. Grav. 51 (2019) no.1, 12. doi:10.1007/s10714-018-2497-7

[86] H. Kim, H. W. Lee and Y. S. Myung, "Equation of state for an interacting holographic dark energy model," Phys. Lett. B 632 (2006), 605-609

[87] P. Avelino and H. da Silva, "Effective dark energy equation of state in interacting dark energy models," Phys. Lett. B 714 (2012), 6-10

[88] W. Zimdahl, "Interacting dark energy and cosmological equations of state," Int. J. Mod. Phys. D 14 (2005), 2319-2326

[89] M. Jamil and M. A. Rashid, "Interacting Dark Energy with Inhomogeneous Equation of State," Eur. Phys. J. C 56 (2008), 429-434

[90] W. Yang, N. Banerjee and S. Pan, "Constraining a dark matter and dark energy interaction scenario with a dynamical equation of state," Phys. Rev. D 95 (2017) no.12, 123527

[91] A. Mukherjee, "Reconstruction of interaction rate in Holographic dark energy," JCAP 11 (2016), 055

[92] A. A. Sen and D. Pavon, "Reconstructing the interaction rate in holographic models of dark energy," Phys. Lett. B 664 (2008) 7 doi:10.1016/j.physletb.2008.04.055 
[93] W. Zimdahl and D. Pavon, "Interacting holographic dark energy" Class. Quant. Grav. 24 (2007) 5461 doi:10.1088/0264-9381/24/22/011 [astro-ph/0606555].

[94] M. Betoule et al. [SDSS Collaboration], "Improved cosmological constraints from a joint analysis of the SDSS-II and SNLS supernova samples," Astron. Astrophys. 568 (2014) A22 doi:10.1051/0004-6361/201423413

[95] C. Zhang, H. Zhang, S. Yuan, T. J. Zhang and Y. C. Sun, "Four new observational $H(z)$ data from luminous red galaxies in the Sloan Digital Sky Survey data release seven," Res. Astron. Astrophys. 14 (2014) no.10, 1221 doi:10.1088/1674-4527/14/10/002; J. Simon, L. Verde and R. Jimenez, "Constraints on the redshift dependence of the dark energy potential," Phys. Rev. D 71 (2005) 123001 doi:10.1103/PhysRevD.71.123001; M. Moresco, L. Verde, L. Pozzetti, R. Jimenez and A. Cimatti, "New constraints on cosmological parameters and neutrino properties using the expansion rate of the Universe to z 1.75," JCAP 1207 (2012) 053 doi:10.1088/1475-7516/2012/07/053; M. Moresco et al., "A $6 \%$ measurement of the Hubble parameter at $z \sim 0.45$ : direct evidence of the epoch of cosmic re-acceleration," JCAP 1605 (2016) 014 doi:10.1088/1475-7516/2016/05/014; A. L. Ratsimbazafy, S. I. Loubser, S. M. Crawford, C. M. Cress, B. A. Bassett, R. C. Nichol and P. Väisänen, "Age-dating Luminous Red Galaxies observed with the Southern African Large Telescope," Mon. Not. Roy. Astron. Soc. 467 (2017) no.3, 3239 doi:10.1093/mnras/stx301; M. Moresco, "Raising the bar: new constraints on the Hubble parameter with cosmic chronometers at $z \sim 2$," Mon. Not. Roy. Astron. Soc. 450 (2015) no.1, L16 doi:10.1093/mnrasl/slv037.

[96] S. Alam et al. [BOSS Collaboration], "The clustering of galaxies in the completed SDSSIII Baryon Oscillation Spectroscopic Survey: cosmological analysis of the DR12 galaxy sample," Mon. Not. Roy. Astron. Soc. 470 (2017) no.3, 2617 doi:10.1093/mnras/stx721

[97] A. Font-Ribera et al. [BOSS Collaboration], "Quasar-Lyman $\alpha$ Forest Cross-Correlation from BOSS DR11 : Baryon Acoustic Oscillations," JCAP 1405 (2014) 027 doi:10.1088/1475-7516/2014/05/027

[98] S. Vagnozzi, L. Visinelli, O. Mena and D. F. Mota, Vagnozzi, "Do we have any hope of detecting scattering between dark energy and baryons through cosmology?." Mon. Not. R. Astron. Soc 493 (2020) 1, 1139. arXiv:1911.12374.

[99] E. Calabrese, R. Putter, D. Huterer, E. V. Linder and A. Melchiorri, "Future CMB constraints on early, cold, or stressed dark energy," Phys. Rev. D83, 023011(2011).

[100] S. DeDeo, R. R. Caldwell and P. J. Steinhardt, "Effects of the sound speed of quintessence on the microwave background and large scale structure," Phys. Rev. D 67, 103509 (2003).

[101] D. F. Mota and D. J. Shaw, "Evading Equivalence Principle Violations, Cosmological and other Experimental Constraints in Scalar Field Theories with a Strong Coupling to Matter," Phys. Rev. D 75, 063501 (2007).

[102] J. Q. Xia, Y. F. Cai, T. T. Qiu, G. B. Zhao and X. Zhang, "Constraints on the Sound Speed of Dynamical Dark Energy," Int. J. Mod. Phys. D 17, 1229 (2008). 
[103] M. Archidiacono, L. Lopez-Honorez and O. Mena, "Current constraints on early and stressed dark energy models and future $21 \mathrm{~cm}$ perspectives," Phys. Rev. D 90, no. 12, 123016 (2014).

[104] S. Hannestad, "Constraints on the sound speed of dark energy," Phys. Rev. D 71 (2005) 103519 doi:10.1103/PhysRevD.71.103519 [astro-ph/0504017].

[105] R. de Putter, D. Huterer and E. V. Linder, "Measuring the Speed of Dark: Detecting Dark Energy Perturbations," Phys. Rev. D 81 (2010) 103513 doi:10.1103/PhysRevD.81.103513 arXiv:1002.1311 [astro-ph.CO]].

[106] C. Carbone, O. Mena and L. Verde, "Cosmological Parameters Degeneracies and Non-Gaussian Halo Bias," JCAP 1007 (2010) 020 doi:10.1088/1475-7516/2010/07/020 arXiv:1003.0456 [astro-ph.CO]].

[107] R. C. Nunes, S. Pan and E. N. Saridakis, "New constraints on interacting dark energy from cosmic chronometers," Phys. Rev. D 94 (2016) no.2, 023508

[108] H. Kim, "Brans-Dicke theory as an unified model for dark matter - dark energy," Mon. Not. Roy. Astron. Soc. 364 (2005), 813-822

[109] Y. S. Myung, "Instability of holographic dark energy models," Phys. Lett. B 652 (2007), 223-227

[110] K. Y. Kim, H. W. Lee and Y. S. Myung, "Instability of agegraphic dark energy models," Phys. Lett. B 660 (2008), 118-124

[111] S. Chakraborty, S. Guha and D. Panigrahi, "Evolution of FRW universe in variable modified Chaplygin gas model," arXiv:1906.12185 [gr-qc]].

[112] J. C. Fabris and J. Martin, "Amplification of density perturbations in fluids with negative pressure," Phys. Rev. D 55 (1997), 5205-5207

[113] F. Yu, J. Zhang, J. Lu, W. Wang and Y. Gui, "A more general interacting model of holographic dark energy," Phys. Lett. B 688 (2010), 263-268

[114] B. Wang, Y. g. Gong and E. Abdalla, "Transition of the dark energy equation of state in an interacting holographic dark energy model," Phys. Lett. B 624 (2005), 141-146 doi:10.1016/j.physletb.2005.08.008 